\title{
Scaling properties of net information measures for bound states of spherical model potentials confined with finite barrier ${ }^{\#}$
}

\author{
S H PATIL ${ }^{\mathrm{a}}$ and $\mathrm{K} \mathrm{D} \mathrm{SEN}^{\mathrm{b}, *}$ \\ ${ }^{a}$ Department of Physics, Indian Institute of Technology Bombay, Mumbai 400 076, India \\ ${ }^{\mathrm{b}}$ School of Chemistry, University of Hyderabad, Hyderabad 500 046, India \\ e-mail: sharad@phy.iitb.ac.in; sensc@uohyd.ernet.in
}

\begin{abstract}
Using dimensional analyses, the scaling properties of the Heisenberg uncertainty relationship as well as the various information theoretical uncertainty-like relationships are derived for the bound states corresponding to the superposition of the power potential of the form $V(r)=Z r^{n}+\sum_{i} Z_{i} r^{n_{i}}$, where $Z, Z_{i}, n, n_{i}$ are parameters, in the free state as well as in the additional presence of a spherical penetrable boundary wall located at radius $R$ The uncertainty product and all other net information measures are shown here to depend only on the parameters $\left[s_{i}\right]$ defined by the ratios $Z_{i} / Z^{\left(n_{i}+2\right) /(n+2)}$. Introduction of a finite potential, $V_{c}$ at the radial distance $r \geq R$ results in a complete set of scaling parameters given by $\left[s_{i}, t_{1}, t_{2}\right]$, where $t_{1}$ is given by $R Z^{1 /(n+2)}$ and $t_{2}=V_{c} /(Z)^{2 /(n+2)}$.
\end{abstract}

Keywords. Heisenberg uncertainty; information theory; confined potentials.

\section{Introduction}

The uncertainty relations are the basic properties of quantum mechanics, in particular, we have the Heisenberg uncertainty principle ${ }^{1}$ for the product of the uncertainties in position and momentum,

$$
\begin{aligned}
& \sigma_{x} \sigma_{p} \geq \frac{1}{2} \hbar, \\
& \sigma_{x}^{2}=<(x-<x>)^{2}>, \\
& \sigma_{p}^{2}=<\left(p_{x}-<p_{x}>\right)^{2}>,
\end{aligned}
$$

in terms of Planck's constant. The uncertainty product has many interesting properties for different potentials, for example, the product for bound states in homogeneous, power potentials is independent of the strength of the potentials. While the uncertainty principle is by definition expressed in terms of the quantum mechanical expectation values, ${ }^{2}$ there exist quite a few other interesting uncertainty-like relationships expressed in terms of the information theoretical measures defined in terms of the probability density alone. Here we will consider some general properties for the bound states in superpositions of power potentials with a finite barrier. It is observed that the dimensionality and scaling properties lead to interesting properties of the uncertainty product, and densities with implications for entropies and information. The aim of such a study is to ascertain how the parameters in the potential describe the

\footnotetext{
\#Dedicated to Prof. N Sathyamurthy on his 60th birthday

*For correspondence
}

various information-theoretical uncertainty-like measures expressed through the electron probability density as the key parameter. We would like to mention that the work presented here forms part of a more general review article in a monograph on statistical complexity ${ }^{3}$ which summarizes the various scaling properties of the uncertainty-like relationships corresponding to a large number of spherical potentials studied by us during the past five years. The work on the superpositions of the power potentials with a finite potential barrier is being presented here in a regular journal for the first time.

\section{Heisenberg uncertainty relations}

Here we analyse some dimensionality properties and their implications for the uncertainty relations for the bound states in superpositions of power potentials with a finite barrier. Similar analysis for a host of spherical model potentials have been reported earlier. ${ }^{4}$ However, the present work provides the first example of a very general class of spherical potential with a finite barrier.

\subsection{Superpositions of power potentials with a finite barrier}

Consider a potential of the form

$V(r)=\left[Z r^{n}+\sum_{i} Z_{i} r^{n_{i}}\right] \theta(R-r)+V_{c} \theta(r-R)$, 
where $Z, Z_{i}, n, n_{i}, R, V_{c}$ are parameters $\left(n, n_{i}\right.$ may not be integers) with $\theta$ being the Heaviside theta function, ${ }^{5}$ in which there are bound states for a particle of mass $M$. Specifically, we have

$$
V_{1}(r)=\left[-k r^{2}+\lambda r^{4}\right] \theta(R-r)+V_{c} \theta(r-R)
$$

for a symmetric double well potential, ${ }^{6}$

$$
V_{2}(r)=\left[\frac{1}{2} k r^{2}+\frac{a}{r^{2}}\right] \theta(R-r)+V_{c} \theta(r-R)
$$

for a modified s. h. o., ${ }^{7}$ and

$$
V_{3}(r)=\left[-\frac{Z}{r}+\lambda r\right] \theta(R-r)+V_{c} \theta(r-R)
$$

for a hydrogenic system confined under a linear potential. ${ }^{8}$ The Schrödinger equation for the potential in equation 2 is

$$
\begin{aligned}
-\frac{\hbar^{2}}{2 M} \nabla^{2} \psi & +\left[\left(Z r^{n}+\sum Z_{i} r^{n_{i}}\right) \theta(R-r)\right. \\
& \left.+V_{c} \theta(r-R)\right] \psi=E \psi .
\end{aligned}
$$

\subsection{Dimensionality and uncertainty relations}

The basic dimensional parameters in our Schrödinger equation are $\hbar^{2} / M, Z, Z_{i}, R, V_{c}$. Of these,

$$
\begin{aligned}
s_{i} & =\frac{M}{\hbar^{2}} Z_{i}\left(\frac{\hbar^{2}}{M Z}\right)^{\left(\frac{n_{i}+2}{n+2}\right)}, \quad t_{1}=R\left(M Z / \hbar^{2}\right)^{1 /(n+2)}, \\
t_{2} & =V_{c}\left(M / \hbar^{2}\right)\left(\hbar^{2} / M Z\right)^{2 /(n+2)}
\end{aligned}
$$

are the dimensionless parameters. Now we consider the deviations

$\sigma_{\vec{r}}^{2}=<(\vec{r}-<\vec{r}>)^{2}>, \quad \sigma_{\vec{p}}^{2}=<(\vec{p}-<\vec{p}>)^{2}>$.

For our potential in equation 6 , the dimensionality properties imply that the deviations are of the form

$$
\begin{aligned}
\sigma_{\vec{r}} & =\left(\hbar^{2} / M Z\right)^{1 /(n+2)} g_{1}\left(s_{i}, t_{i}\right), \\
\sigma_{\vec{p}} & =\hbar\left(M Z / \hbar^{2}\right)^{1 /(n+2)} g_{2}\left(s_{i}, t_{i}\right),
\end{aligned}
$$

so that the uncertainty product is

$$
\begin{aligned}
\sigma_{\vec{r}} \sigma_{\vec{p}} & =\hbar g_{1}\left(s_{i}, t_{i}\right) g_{2}\left(s_{i}, t_{i}\right), \\
s_{i} & =\frac{M}{\hbar^{2}} Z_{i}\left(\frac{\hbar^{2}}{M Z}\right)^{\left(\frac{n_{i}+2}{n+2}\right)}, \\
t_{1} & =R\left(M Z / \hbar^{2}\right)^{1 /(n+2)}, \\
t_{2} & =V_{c}\left(M / \hbar^{2}\right)\left(\hbar^{2} / M Z\right)^{2 /(n+2)} .
\end{aligned}
$$

This implies that the uncertainty product depends only on the dimensionless parameters $s_{i}, t_{i}$. It may also be noted that the bound state energies are of the form

$$
E=\left(\hbar^{2} / M\right)\left(M Z / \hbar^{2}\right)^{2 /(n+2)} g_{3}\left(s_{i}, t_{i}\right) .
$$

These results follow from just the dimensionality properties of the parameters.

It is interesting to note that one can analyse the minimum uncertainty in terms of the scaled parameters. For example, in the case of a hydrogen atom with a finite barrier potential, one can obtain the minimum uncertainty by keeping $V_{c}$ fixed but varying $R Z$. Alternatively, one can keep $R$ fixed but vary $V_{c}$. These considerations can be extended to the superpositions of power potentials with a finite barrier potential in terms of the scaled variables $s_{i}$ and $t_{i}$.

\section{Scaling properties and entropies}

We will now consider some scaling properties for bound states in a superposition of power potentials, and their implications for Shannon entropy ${ }^{9,10}$ and other properties.

\subsection{Scaling properties}

For the Schrödinger equation in equation 6, the energy $E$ and eigenfunction $\psi$ are functions of the form

$$
\begin{aligned}
& E: E\left(\hbar^{2} / M, Z, Z_{i}, R, V_{c}\right), \\
& \psi: \psi\left(\hbar^{2} / M, Z, Z_{i}, R, V_{c}, r\right) .
\end{aligned}
$$

Multiplying equation 6 by $M / \hbar^{2}$, and introducing a scale transformation

$$
\vec{r}=\lambda \vec{r}^{\prime}
$$

one gets

$$
\begin{aligned}
& -\frac{1}{2} \nabla^{\prime 2} \psi+\left(M / \hbar^{2}\right) \\
& \quad \times\left[Z \lambda^{n+2} r^{\prime n}+\sum Z_{i} \lambda^{n_{i}+2} r^{\prime n_{i}}\right] \theta\left(R / \lambda-r^{\prime}\right) \psi \\
& \quad+\left(M / \hbar^{2}\right) \lambda^{2} V_{c} \theta\left(r^{\prime}-R / \lambda\right) \psi=\left(M / \hbar^{2}\right) \lambda^{2} E \psi
\end{aligned}
$$

Taking

$$
\lambda=\left(\frac{\hbar^{2}}{M Z}\right)^{1 /(n+2)},
$$


equation (14) leads to

$$
\begin{aligned}
& -\frac{1}{2} \nabla^{\prime 2} \psi+\left[r^{\prime n}+\frac{M}{\hbar^{2}} \sum Z_{i}\left(\frac{\hbar^{2}}{M Z}\right)^{\left(\frac{n_{i}+2}{n+2}\right)} r^{\prime n_{i}}\right] \\
& \quad \times \theta\left(R\left(M Z / \hbar^{2}\right)^{1 /(n+2)}-r^{\prime}\right) \psi \\
& \quad+\frac{M}{\hbar^{2}}\left(\hbar^{2} / M Z\right)^{2 /(n+2)} V_{c} \theta\left(r^{\prime}-R\left(M Z / \hbar^{2}\right)^{1 /(n+2)}\right) \psi \\
& \quad=\frac{M}{\hbar^{2}}\left(\frac{\hbar^{2}}{M Z}\right)^{2 /(n+2)} E \psi .
\end{aligned}
$$

Comparing this with equation 6 , we obtain

$$
\begin{aligned}
& E\left(\frac{\hbar^{2}}{M}, Z, Z_{i}, R, V_{c}\right) \\
& \quad=\left(\hbar^{2} / M\right) \lambda^{-2} E\left(1,1, Z_{i} \lambda^{n_{i}+2} M / \hbar^{2}, R / \lambda, V_{c} \lambda^{2} M / \hbar^{2}\right), \\
& \psi\left(\frac{\hbar^{2}}{M}, Z, Z_{i}, R, V_{c}, r\right) \\
& \quad=A \psi\left(1,1, Z_{i} \lambda^{n_{i}+2} M / \hbar^{2}, R / \lambda, V_{c} \lambda^{2} M / \hbar^{2}, r^{\prime}\right), \\
& \quad r=\lambda r^{\prime}, \quad \lambda=\left(\hbar^{2} / M Z\right)^{1 /(n+2)} .
\end{aligned}
$$

Taking $\psi\left(1,1, Z_{i} \lambda^{n_{i}+2} M / \hbar^{2}, R / \lambda, V_{c} \lambda^{2} / \hbar^{2}, r^{\prime}\right)$ to be normalized, the normalization of the wave function $\psi\left(\hbar^{2} / M, Z, Z_{i}, R, V_{c}, r\right)$ leads to

$$
\begin{aligned}
1 & =A^{2} \int\left|\psi\left(1,1, Z_{i} \lambda^{n_{i}+2} M / \hbar^{2}, R / \lambda, V_{c} \lambda^{2} M / \hbar^{2}, r^{\prime}\right)\right|^{2} d^{3} r, \\
& =A^{2} \lambda^{3} \Rightarrow A=\lambda^{-3 / 2}=\left(M Z / \hbar^{2}\right)^{\frac{3}{2(n+2)}}
\end{aligned}
$$

so that

$$
\begin{aligned}
\psi( & \left.\frac{\hbar^{2}}{M}, Z, Z_{i}, R, V_{c}, r\right) \\
& =\lambda^{-3 / 2} \psi\left(1,1, Z_{i} \lambda^{n_{i}+2} M / \hbar^{2}, R / \lambda, V_{c} \lambda^{2} M / \hbar^{2}, r^{\prime}\right), \\
\lambda & =\left(\hbar^{2} / M Z\right)^{1 /(n+2)}, \quad r^{\prime}=r / \lambda .
\end{aligned}
$$

For obtaining the wave function in the momentum space, we take the Fourier transform of the wave function in equation (19), leading to

$$
\begin{aligned}
& f\left(\hbar^{2} / M, Z, Z_{i}, R, V_{c}, p\right) \\
& \quad=\frac{1}{(2 \pi \hbar)^{3 / 2}} \int d^{3} r e^{-i \vec{p} \cdot \vec{r} / \hbar} \psi\left(\hbar^{2} / M, Z, Z_{i}, R, V_{c}, r\right) .
\end{aligned}
$$

Using the relation in equation 19 and changing the integration variable to $r^{\prime}$, we get

$$
\begin{aligned}
& f\left(\hbar^{2} / M, Z, Z_{i}, R, V_{c}, p\right) \\
& \quad=\lambda^{3 / 2} f\left(1,1, Z_{i} \lambda^{n_{i}+2} M / \hbar^{2}, R / \lambda, V_{c} \lambda^{2} M / \hbar^{2}, p^{\prime}\right), \\
& \lambda=\left(\hbar^{2} / M Z\right)^{1 /(n+2)}, \quad p^{\prime}=\lambda p .
\end{aligned}
$$

From the relations in equations 19 and 21, one has for the corresponding position and momentum densities,

$\rho\left(\hbar^{2} / M, Z, Z_{i}, R, V_{c}, r\right)=\lambda^{-3} \rho\left(1,1, s_{i}, t_{1}, t_{2}, r^{\prime}\right)$,

$\gamma\left(\hbar^{2} / M, Z, Z_{i}, R, V_{c}, p\right)=\lambda^{3} \gamma\left(1,1, s_{i}, t_{1}, t_{2}, p^{\prime}\right)$,

$\lambda=\left(\hbar^{2} / M Z\right)^{1 /(n+2)}, \quad s_{i}=\lambda^{n_{i}+2} M Z_{i} / \hbar^{2}, \quad t_{1}=R / \lambda$

$t_{2}=V_{c} \lambda^{2} M / \hbar^{2} \quad r^{\prime}=r / \lambda, \quad p^{\prime}=\lambda p$,

with $s_{i}, t_{i}$ being the scaled parameters.

\subsection{Shannon entropy sum}

The Shannon entropies ${ }^{9}$ in the position space and momentum space, are

$$
\begin{aligned}
& S_{r}=-\int \rho(r)[\ln \rho(r)] d^{3} r, \\
& S_{p}=-\int \gamma(p)[\ln \gamma(p)] d^{3} p .
\end{aligned}
$$

Using the relations in equation 22 , we get for these entropies

$S_{r}\left(\hbar^{2} / M, Z, Z_{i}, R, V_{c}\right)=3 \ln \lambda+S_{r}\left(1,1, s_{i}, t_{1}, t_{2}\right)$, $S_{p}\left(\hbar^{2} / M, Z, Z_{i}, R, V_{c}\right)=-3 \ln \lambda+S_{p}\left(1,1, s_{i}, t_{1}, t_{2}\right)$,

which imply that the Shannon entropy sum $S_{T}=S_{r}+$ $S_{p}$ satisfies the relation

$$
\begin{aligned}
& S_{T}\left(\hbar^{2} / M, Z, Z_{i}, R, V_{c}\right)=S_{T}\left(1,1, s_{i}, t_{1}, t_{2}\right), \\
& s_{i}=\frac{M}{\hbar^{2}} Z_{i}\left(\frac{\hbar^{2}}{M Z}\right)^{\left(\frac{n_{i}+2}{n+2}\right)}, \quad t_{1}=R\left(M Z / \hbar^{2}\right)^{1 /(n+2),} \\
& t_{2}=V_{c}\left(M / \hbar^{2}\right)\left(\hbar^{2} / M Z\right)^{2 /(n+2)} .
\end{aligned}
$$

Therefore, for given values of the parameters $Z, Z_{i}, R, V_{c}$, the Shannon entropy sum depends only on $Z_{i} / Z^{\left(n_{i}+2\right) /(n+2)}, t_{1}, t_{2}$.

\subsection{Fisher information}

The Fisher information measures ${ }^{11-14}$ for position and momentum are

$$
I_{r}=\int \frac{[\vec{\nabla} \rho(r)]^{2}}{\rho(r)} d^{3} r, \quad I_{p}=\int \frac{[\vec{\nabla} \gamma(p)]^{2}}{\gamma(p)} d^{3} p .
$$


Using the relations in equation 22 , one obtains

$$
\begin{aligned}
& I_{r}\left(\hbar^{2} / M, Z, Z_{i}, R, V_{c}\right)=\frac{1}{\lambda^{2}} I_{r}\left(1,1, s_{i}, t_{1}, t_{2}\right), \\
& I_{p}\left(\hbar^{2} / M, Z, Z_{i}, R, V_{c}\right)=\lambda^{2} I_{p}\left(1,1, s_{i}, t_{1}, t_{2}\right) .
\end{aligned}
$$

which together imply that the Fisher information product $I_{r} I_{p}$ satisfies the relation

$$
\begin{aligned}
& I_{r p}\left(\hbar^{2} / M, Z, Z_{i}, R, V_{c}\right)=I_{r p}\left(1,1, s_{i}, t_{1}, t_{2}\right), \\
& I_{r p}=I_{r} I_{p}, \\
& s_{i}=\frac{M}{\hbar^{2}} Z_{i}\left(\frac{\hbar^{2}}{M Z}\right)^{\left(\frac{n_{i}+2}{n+2}\right)}, t_{1}=R\left(M Z / \hbar^{2}\right)^{1 /(n+2)}, \\
& t_{2}=V_{c}\left(M / \hbar^{2}\right)\left(\hbar^{2} / M Z\right)^{2 /(n+2)} .
\end{aligned}
$$

Here, for given values of the parameters $Z, Z_{i}, R, V_{c}$, the Fisher information product depends only on $Z_{i} / Z^{\left(n_{i}+2\right) /(n+2)}, t_{1}, t_{2}$.

\subsection{Rényi entropy}

The Rényi entropies ${ }^{15}$ in position and momentum spaces are

$$
\begin{aligned}
H_{\alpha}^{(r)} & =\frac{1}{1-\alpha} \ln \int[\rho(r)]^{\alpha} d^{3} r, \\
H_{\alpha}^{(p)} & =\frac{1}{1-\alpha} \ln \int[\gamma(p)]^{\alpha} d^{3} p .
\end{aligned}
$$

With the relations in equation 22, we get for these entropies,

$$
\begin{aligned}
& H_{\alpha}^{(r)}\left(\hbar^{2} / M, Z, Z_{i}, R, V_{c}\right)=3 \ln \lambda+H_{\alpha}^{(r)}\left(1,1, s_{i}, t_{1}, t_{2}\right), \\
& H_{\alpha}^{(p)}\left(\hbar^{2} / M, Z, Z_{i}, R, V_{c}\right) \\
& \quad=-3 \ln \lambda+H_{\alpha}^{(p)}\left(1,1, s_{i}, t_{1}, t_{2}\right),
\end{aligned}
$$

which imply that the Rényi entropy sum $H_{\alpha}^{(T)}=H_{\alpha}^{(r)}+$ $H_{\alpha}^{(p)}$ satisfies the relation

$$
\begin{aligned}
& H_{\alpha}^{(T)}\left(\frac{\hbar^{2}}{M}, Z, Z_{i}, R, V_{c}\right)=H_{\alpha}^{(T)}\left(1,1, s_{i}, t_{1}, t_{2}\right), \\
& s_{i}=\frac{M}{\hbar^{2}} Z_{i}\left(\frac{\hbar^{2}}{M Z}\right)^{\left(\frac{n_{i}+2}{n+2}\right)}, t_{1}=R\left(M Z / \hbar^{2}\right)^{1 /(n+2)}, \\
& t_{2}=V_{c}\left(M / \hbar^{2}\right)\left(\hbar^{2} / M Z\right)^{2 /(n+2)} .
\end{aligned}
$$

Therefore, as in other cases, for given values of the parameters $Z, Z_{i}, R, V_{c}$, the Rényi entropy sum depends on $Z_{i} / Z^{\left(n_{i}+2\right) /(n+2)}, t_{1}, t_{2}$.

\subsection{Onicescu energies}

The Onicescu energies ${ }^{17}$ in position and momentum spaces are

$$
E_{r}=\int[\rho(r)]^{2} d^{3} r, \quad E_{p}=\int[\gamma(p)]^{2} d^{3} p .
$$

Using the relations in equation 22 , we get

$$
\begin{aligned}
& E_{r}\left(\hbar^{2} / M, Z, Z_{i}, R, V_{c}\right)=\frac{1}{\lambda^{3}} E_{r}\left(1,1, s_{i}, t_{1}, t_{2}\right), \\
& E_{p}\left(\hbar^{2} / M, Z, Z_{i}, R, V_{c}\right)=\lambda^{3} E_{p}\left(1,1, s_{i}, t_{1}, t_{2}\right),
\end{aligned}
$$

which imply that the Onicescu energy product $E_{r p}=$ $E_{r} E_{p}$ satisfies the relation

$$
\begin{aligned}
& E_{r p}\left(\hbar^{2} / M, Z, Z_{i}, R, V_{c}\right)=E_{r p}\left(1,1, s_{i}, t_{1}, t_{2}\right), \\
& s_{i}=\frac{M}{\hbar^{2}} Z_{i}\left(\frac{\hbar^{2}}{M Z}\right)^{\left(\frac{n_{i}+2}{n+2}\right)}, t_{1}=R\left(M Z / \hbar^{2}\right)^{1 /(n+2)}, \\
& t_{2}=V_{c}\left(M / \hbar^{2}\right)\left(\hbar^{2} / M Z\right)^{2 /(n+2)} .
\end{aligned}
$$

In this case also, for given values of the parameters $Z, Z_{i}, R, V_{c}$, the Onicescu energy product depends on $Z_{i} / Z^{\left(n_{i}+2\right) /(n+2)}, t_{1}, t_{2}$.

\subsection{Tsallis entropy}

The Tsallis entropies ${ }^{16}$ in position and momentum spaces are

$$
\begin{aligned}
& T_{r}=\frac{1}{q-1}\left[1-\int[\rho(r)]^{q} d^{3} r\right], \\
& T_{p}=\frac{1}{m-1}\left[1-\int[\gamma(p)]^{m} d^{3} p\right], \quad \frac{1}{q}+\frac{1}{m}=2 .
\end{aligned}
$$

We consider the integral terms

$$
\begin{aligned}
& J_{r}\left(\hbar^{2} / M, Z, Z_{i}, R, V_{c}\right)=\int[\rho(r)]^{q} d^{3} r, \\
& J_{p}\left(\hbar^{2} / M, Z, Z_{i}, R, V_{c}\right)=\int[\gamma(p)]^{m} d^{3} p .
\end{aligned}
$$

Using the relations in equation 22 , we get

$$
\begin{aligned}
& J_{r}\left(\hbar^{2} / M, Z, Z_{i}, R, V_{c}\right)=\lambda^{3-3 q} J_{r}\left(1,1, s_{i}, t_{1}, t_{2}\right), \\
& J_{p}\left(\hbar^{2} / M, Z, Z_{i}, R, V_{c}\right)=\lambda^{3 m-3} J_{p}\left(1,1, s_{i}, t_{1}, t_{2}\right) .
\end{aligned}
$$


Then one obtains for the ratio,

$$
\begin{aligned}
& J_{p / r}\left(\hbar^{2} / M, Z, Z_{i}, R, V_{c}\right)=J_{p / r}\left(1,1, s_{i}, t_{1}, t_{2}\right), \\
& J_{p / r}=\frac{J_{p}^{1 / 2 m}}{J_{r}^{1 / 2 q}}, \quad \frac{1}{m}+\frac{1}{q}=2, \\
& s_{i}=\frac{M}{\hbar^{2}} Z_{i}\left(\frac{\hbar^{2}}{M Z}\right)^{\left(\frac{n_{i}+2}{n+2}\right)}, \quad t_{1}=R\left(M Z / \hbar^{2}\right)^{1 /(n+2)}, \\
& t_{2}=V_{c}\left(M / \hbar^{2}\right)\left(\hbar^{2} / M Z\right)^{2 /(n+2)} .
\end{aligned}
$$

Therefore in this case also, for given values of the parameters $Z, Z_{i}, R, V_{c}$, the ratio of Tsallis entropies depends on $Z_{i} / Z^{\left(n_{i}+2\right) /(n+2)}, t_{1}, t_{2}$.

\section{Summary}

For the bound states corresponding to the superposition of the power potential of the form $V(r)=Z r^{n}+$ $\sum_{i} Z_{i} r^{n_{i}}$, where $Z, Z_{i}, n, n_{i}$ are parameters, in the free state as well as in the additional presence of a spherical penetrable boundary wall located at radius $R$, the quantum mechanical uncertainty product and all other net information measures are shown here to depend only on the parameters $\left[s_{i}\right]$ defined by the ratios $Z_{i} / Z^{\left(n_{i}+2\right) /(n+2)}$. Further, we have shown that the introduction of a finite potential, $V_{c}$ at the radial distance $r \geq R$ results in a complete set of scaling parameters given by $\left[s_{i}, t_{1}, t_{2}\right]$, where $t_{1}$ is given by $R Z^{1 /(n+2)}$ and $t_{2}=V_{c} /(Z)^{2 /(n+2)}$. It is significant to note that the uncertainty like measures scale with the finite barrier as given by the parameter $t_{2}$.

\section{Acknowledgements}

One of the authors, SHP acknowledges support from AICTE (All India Council for Technical Education) as Emeritus Fellow. KDS is grateful to the Department of Science and Technology for the award of JC Bose National Fellowship during 2009-2014.

\section{References}

1. (a) Heisenberg W 1927 Z. Phys. 43 172; (b) Kennard E H 1927 Z. Phys. 44326

2. (a) Tsapline B 1970 Chem. Phys. Lett. 6 596; (b) Majernik V and Richterek L 1997 J. Phys. A: Math. Gen. 30 L49; (c) Grypeoos M E, Koutroulos C G, Oyewumi K J and Petridou Th. 2004 J. Phys A: Math. Gen. 37 7895; (d) Kuo C 2005 Ann. Phys. 316 431; (e) Qiang W -C and Dong S -H 2006 J. Phys. A 39 8663. [ All these works report extensive numerical tests of the square of Heisenberg product, $<r^{2}><p^{2}>$, in atomic systems.

3. Sen K D (Ed.) 2011 Statistical complexity: Applications in electronic structure (UK: Springer) Chapter 8

4. (a) Sen K D and Katriel J 2006 J. Chem. Phys. 125 074117; (b) Patil S H and Sen K D 2007 Phys. Lett. A 362 109; (c) Patil S H and Sen K D 2007 Phys. Lett. A 370 354; (d) Patil S H, Sen K D, Watson N A and Montgomery H E Jr, 2007 J. Phys. B 40 2147; (e) González-Férez R, Dehesa J S, Patil S H, Sen K D 2009 Physica A 3884919

5. Abramowitz M and Stegun I A 1972 Handbook of mathematical functions with formulas (Government Printing Office Washington DC) pp 1020

6. Banerjee K and Bhatnagar S P 1978 Phys. Rev. D 184767

7. Kratzer A 1920 Z. Phys. 3289

8. Dullemond C 1978 Phys. Rev. D 78574

9. Shannon C E 1948 Bell Syst. Tech. 27 379; 1948 Bell Syst. Tech. 27623

10. Bialynicki-Birula I and Mycielski J 1975 Сотmun. Math. Phys. 44129

11. Fisher R A 1925 Proc. Camb. Philos. Soc. 22700

12. Frieden B R 2004 Science from fisher information (Cambridge University Press)

13. Rao C R 1965 Linear statistical interference and its applications (New York: Wiley)

14. Sears S B 1980 Applications of information theory in chemical physics Ph.D. thesis (University of North Carolina at Chapel Hill)

15. Rényi A 1960 Some fundamental questions of information theory, MTA III Oszt. Közl. 10 251; 1960 On measures of information and entropy, Proccedings of the Fourth Berkeley Symposium on Mathematics, Statistics and Probability (Berkeley University Press, Berkeley CA) pp 547; 1970 Probability theory (North-Holland, Amsterdam)

16. Tsallis C 1988 J. Stat. Phys. 52479

17. Onicescu O 1966 CR Acad. Sci. Paris A 26325 